the terms cirro-stratus and strato-cirrus. Finally the terms cumulo-stratus and cirro-stratus are sorcly nceded for varietics of clouds intermediate between the class I have described and the cumulus and cirrus types, if any part of Ioward's terminology is to be left to us at all.

It would be a pity that that terminology, lucid and expressive, should perish, merely because, to a few minds, the originator of a system must needs appear infallible, and his classification perfect as Minerva when issuing from the head of Jupiter. I think that Luke Ioward would bave been the last to put forward such a claim.

June 8

W. Clemext Ley

\section{The Motion of Fluids}

Prof. Reynolds, in the course of his review (NATURE, vol. xxi. p. 342) of my book on the above subject, cites two instances in which I have been guilty of what he considers loose and vague reasoning. I would ask space for a few remarks on the points in question.

To take the more important matter first, Frof. Keynolds says, apropos of a certain proof of the velocity-potential theorem given in Art. 23 :-

"Mr. Lamb has offered a proof of this now historic theorem, which, if judged by the space it occupies, should be much simpler than the acknowledged proofs of Cauchy and Stokes. As no authority is cited, it would appear that this proof is here given for the first time. If so, the author has done himself great injustice in not examining or explaining his reasoning morc closely. For, as it stands, it suggests the idea that he has ignored the fact that $d x, d y, d z$, on the left of his equation, are integrals through a finite time, and hence, inasmuch as he has giren no reason to the contrary, may be of a different order of magnitude from their initial values, $d a, d b, d c$, which appear on the right of his equation. If this is not so it is a peculiarity of the motion of continuous fluicl, and needs establishing; otherwise we might infer that two people who had once shaken hands could never after be so much as a mile apart.".

Prof. Reynolds, who himself strongly recommends the careful study of "work from the master's hand," will hardly take it amiss if I ask him to turn to the proofs which he justly cites as classical, and to notice that they contain, onc of them (Cauchy's) in exactly the same form, the other in a form which is mathematically equivalent, the very assumption which he here calls in question. The assumption is in fact nothing more than a tacit limitation, which is made at the very outset of the subject, as to the class of motions which are proposed for study. In the "Eulerian" method it is implied that the first derivatives of the component velocitics $z, v, w$ with respect to the co-ordinates $x, y, z$ are to be everywhere and always finite throughout the motion considerel ; in the "I.agrangian" method the corresponding, and equivalent, assumption is that the derivatives $\frac{d x}{d a}, \frac{d x}{d b}, \frac{d x}{d c}$, \&c, and also

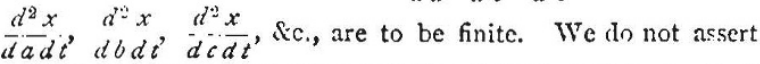
that these are universal characteristics of fluid motion, for it is casy to imagine cases in which they are violated; we merely exclude such cascs ab initio from the scope of our investigations. But, in onc form or another, these fundamental limilations are, from the point of view of analytical hydrodynamics, unavoic!able; they are made implicitly every time we write down the equations of motion, and it is therefore not surprising that they should be found to be essentially involvel, not only in the proof which Prof. Reynolds on this account criticises, but in every other proof of the velocity-potential theorem which has yet been propounded.

I bave only to add that the proof in question is, and professes to be, merely a very obvious corollary to $H$. Weler's transforunation of the Lagrangian equations.

The other passage of Prof. Reynolds's review which I wish to notice is as follows :-

"There is a considerable amount of vagueness altencing the author's use of the term particle. Having rightly lefined fluids as being such 'that the properties of the smallest portions into which we can conceive them divided are the same as those of the substance in bulk,' he proceeds to reason about a particle as though it were a discretc quantity, the position of which is defined by some point, thus ignoring the fact that, accorling to his definftion, the same particle of fluid may at one time be a sphcre, at another a filament of indefinitc length, or a sheet of inclefinite breadth. This ragueness anpears to have led him into crror in Art. II."

$A$ rood deal of this criticism is, I think, met by the remarks already made. In a fluid moving subject to the conditions I have stated, only finite changes of shape can be produced in a moving element within a finite time.

Prof. Reynolds does not indicate the precise nature of the "error" which he finds in Art. 11. After a careful reconsideration, the argument of that article appears to me to be sound ; but I an free to confess that it is not stated with all the clearnes: desirable, and that the article is further disfigured by an unfortunate clerical error in the foot-note, where " $u= \pm \sqrt{x}$ " should be read for " $u= \pm x$."

Adelaide, March 30

\section{On the Physical Aspccts of the Vortex-Atom Theory}

WILL any charitable person explain a difficulty which I (and other non-mathematical people) have encountered when seeking to understand and be satisfied with this theory?

The only proof of those properties of vortex rings which match the physical properties of atons that $I$ have met with is that in Bésant's "IIydromechanics"; and is bascd on the initial.coordinate method.

Now it scems to me that this method assumes what is equivalent to the permanence of the vortex filament; so that in proving the latter by use of this system of co-ordinates we may be merely arguing in a circle.

For it assumes that if initially we have any infinitesimal tetrabedron $\delta a . \delta \beta . \delta \gamma$, then after the finite time, $t$, this will still form a tetrahedron $\delta x . \delta y^{\prime} . \delta z$.

Now I cannot see that one can assume this; that-to use the words in a late article of NATURE-. "if two people have once shaken hands they can never be 100 miles apart."

And this inseparability of the particles of a fluid thus assumed bears a very close relation to the permanence of the vortex filament which we wish to prove.

W. L.

Cheltenham, May 29

[It appears to us that our correspondent here confuses between the pernianence of any fluid filament and the permanence of the vortex character of the filament. The assumption that every filament remains continuous cannot be said to be equivalent to assuming that the direction of the filament at every point remains coincident with the axis of rotation of its constituent elements at that point, which is what Helmboltz has taught us.-ED.]

\section{The Aurora Borealis and its Colours}

W'ITIr regard to Drs. De La Rue and Müller's paper on the Aurora (NATURE, vol, xxii. p. 33) there is still a point I should like to sec explained. Is it considered by physicists that in electric discharges similarity of colour is sufficient to indicate similarity of constitution, cven when their spectra are quite unlike? The paper, together with the reply to Prof. Suyth, certainly seems to imply this; though I have not previously seen it stated to be the case.

With regard to the red part of aurorx, so far as my observations indicate its position, they show it to be above the greenish part in the aurorie seen here; though according to Weyprecht's ubservations, it is below the green in the Arctic regions.

Sunderlank, June 9

T. W. BACKHOUSE

\section{A New Audiphone}

FURTHER experiments on the timbre of musical instruments as rendered by the audiphone have led me to the selcction of the following as a distinct improvement on the birchwood veneer, both for musical purposes and also for ordinary conversation. It has the same advantage as my previous form in not reçuiring to be beld by the hand, it costs nothing, and requires no making. Tirtic a shect of stiff brown payer ahout II $\times$ i 5 inchcs, the paper being such as is ordinarily used for making up heavy parcels. Put the ends together, the midile forming a loop, and hold the ends lictween the tecth. The paper must be pretty stiff, as the loop minst stand out round and full, and of course the paper must be without fulds or creases.

Museum Street, Warrington

\section{Crystal-Ice}

I. reference to the "crystal ice" proposed hy Dr. Calintarients, of Scarborough, f,r skating upon with ordinary skates, 